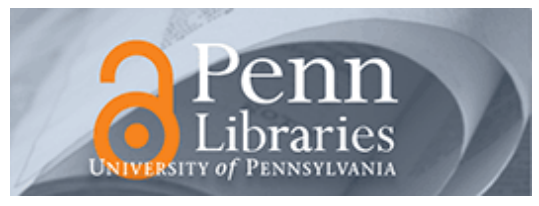

University of Pennsylvania ScholarlyCommons

March 2006

\title{
Interfacial in situ polymerization of single wall carbon nanotube/ nylon 6,6 nanocomposites
}

\author{
Reto Haggenmueller \\ University of Pennsylvania \\ Fangming Du \\ University of Pennsylvania \\ John E. Fischer \\ UNiversity of Pennsylvania \\ Karen I. Winey \\ University of Pennsylvania, winey@lrsm.upenn.edu
}

Follow this and additional works at: https://repository.upenn.edu/mse_papers

\section{Recommended Citation}

Haggenmueller, R., Du, F., Fischer, J. E., \& Winey, K. I. (2006). Interfacial in situ polymerization of single wall carbon nanotube/nylon 6,6 nanocomposites. Retrieved from https://repository.upenn.edu/

mse_papers/106

Postprint version. Published in Polymer, Volume 47, Issue 7, 2006, pages 2381-2388.

Publisher URL: http://dx.doi.org/10.1016/j.polymer.2006.01.087

This paper is posted at ScholarlyCommons. https://repository.upenn.edu/mse_papers/106

For more information, please contact repository@pobox.upenn.edu. 


\title{
Interfacial in situ polymerization of single wall carbon nanotube/nylon 6,6 nanocomposites
}

\begin{abstract}
An interfacial polymerization method for nylon 6,6 was adapted to produce nanocomposites with single wall carbon nanotubes (SWNT) via in situ polymerization. SWNT were incorporated in purified, functionalized or surfactant stabilized forms. The functionalization of SWNT was characterized by FTIR, Raman spectroscopy and TGA and the SWNT dispersion was characterized by optical microscopy before and after the in situ polymerization. SWNT functionalization and surfactant stabilization improved the nanotube dispersion in solvents but only functionalized SWNT showed a good dispersion in composites, whereas purified and surfactant stabilized SWNT resulted in poor dispersion and nanotube agglomeration. Weak shear flow induced SWNT flocculation in these nanocomposites. The electrical and mechanical properties of the SWNT/nylon nanocomposites are briefly discussed in terms of SWNT loading, dispersion, length and type of functionalization.
\end{abstract}

\section{Keywords}

nanocomposite, single wall carbon nanotube, in situ polymerization

\section{Comments}

Postprint version. Published in Polymer, Volume 47, Issue 7, 2006, pages 2381-2388.

Publisher URL: http://dx.doi.org/10.1016/j.polymer.2006.01.087 


\title{
Interfacial In Situ Polymerization of Single Walled Carbon Nanotube / Nylon 6,6 Nanocomposites
}

\author{
Reto Haggenmueller ${ }^{\mathrm{a}}$, Fangming Du ${ }^{\mathrm{b}}$, John E. Fischer ${ }^{\mathrm{a}}$, and Karen I. Winey ${ }^{\mathrm{a}, *}$ \\ ${ }^{a}$ University of Pennsylvania, Department of Materials Science and Engineering, \\ Philadelphia, PA 19104-6272, USA \\ ${ }^{\mathrm{b}}$ University of Pennsylvania, Department of Chemical and Biomolecular Engineering, \\ Philadelphia, PA 19104-6393, USA
}

*Corresponding author. Tel.: + 1215 8980593; fax: + 12155732128 .

E-mail address: winey@seas.upenn.edu (K. I. Winey)

Submitted to Polymer on January 23, 2006 


\begin{abstract}
An interfacial polymerization method for nylon 6,6 was adapted to produce nanocomposites with single walled carbon nanotubes (SWNT) via in situ polymerization. SWNT were incorporated in purified, functionalized or surfactant stabilized forms. The functionalization of SWNT was characterized by FTIR, Raman spectroscopy and TGA and the SWNT dispersion was characterized by optical microscopy before and after the in situ polymerization. SWNT functionalization and surfactant stabilization improved the nanotube dispersion in solvents but only functionalized SWNT showed a good dispersion in composites, whereas purified and surfactant stabilized SWNT resulted in poor dispersion and nanotube agglomeration. Weak shear flow induced SWNT flocculation in these nanocomposites. The electrical and mechanical properties of the SWNT/nylon nanocomposites are briefly discussed in terms of SWNT loading, dispersion, length and type of functionalization.
\end{abstract}

Keywords: Nanocomposite, Single walled carbon nanotube, in situ polymerization. 


\section{Introduction}

Single wall carbon nanotubes (SWNT) are considered promising fillers in nanocomposites due to their exceptional mechanical, electrical, and thermal properties and their large aspect ratio, all of which can lead to significantly improved composite performance. The fabrication of SWNT / polymer nanocomposites has been achieved by the use of several different fabrication methods that combine various polymer matrix systems with carbon nanotubes. Solution processing methods are available if the polymer is soluble in a solvent that can suspend nanotubes, enabling the mixing of polymer and nanotubes in the solvent [1-4]. Melt compounding incorporates the nanotubes into a molten thermoplastic polymer that is mechanically sheared in a compounder [5-9]. Nanotubes can be added to this polymer melt in the compounder dry or suspended in a solvent to achieve good dispersion $[10,11]$. In situ polymerization methods offer the possibility to incorporate SWNT into polymer matrixes while preserving the nanotube dispersion initially found in the reaction medium containing the monomers [12-16].

In any of these fabrication methods, SWNT that are well dispersed in solvents (including monomers) prior to composite fabrication facilitate good SWNT dispersion in the subsequent composites. This can be achieved with the aid of surfactants or functional groups that are self-assembled or covalently attached to the nanotube surface, respectively [17-20]. Well-dispersed SWNT exist as small bundles or individual nanotubes. Incorporation of functionalized nanotubes is preferably done by the use of a solvent processing method or an in situ polymerization to preserve the superior nanotube dispersion.

Nylon 6,6, a commercially important thermoplastic, cannot be readily solvent processed with nanotubes because nylon 6,6 is soluble in only a few solvents that either do not suspend nanotubes (e.g. formic acid) or may even damage nanotubes (e.g. sulfuric acid). Melt compounding can be used, but the melt viscosity of nylon 6,6 is rather low, resulting in small shear forces and poor SWNT dispersion when dry nanotubes are added. We previously used a melt compounder to combine SWNT suspensions using HDPE [11], but this process cannot be applied to nylon 6,6 because the processing temperature $\left(\sim 270^{\circ} \mathrm{C}\right)$ is well above the boiling temperature of suitable solvents to suspend SWNT. 
Here we present an interfacial in situ polymerization method for SWNT / nylon 6,6 nanocomposites that can be used with a variety of SWNT types. Based on the familiar "nylon rope trick", this step growth polymerization method incorporates both an organic and an aqueous phase, each carrying one of the two highly reactive monomers. The polymerization takes place at the interface between the two immiscible organic and aqueous phases where the monomers meet and rapidly react. Thus, nanotubes can be suspended in either phase, allowing the use of functionalized nanotubes that prefer either an aqueous or an organic solvent environment. The initial dispersion of the nanotubes in suspension is preserved in the resulting nanocomposites. Here, SWNT were incorporated into nylon 6,6 nanocomposites from suspensions of purified SWNT, surfactant-assisted suspensions, or suspensions of SWNT functionalized with short alkyl chains, to study the effect of the nanotube dispersion method. The electrical and mechanical properties of the SWNT / nylon 6,6 nanocomposites are also recorded.

\section{Experimental Methods}

SWNT Purification and Functionalization: SWNT were synthesized by the highpressure carbon monoxide method (HiPco, Rice University) [21]. Nanotube suspensions were made with purified, functionalized and surfactant suspended SWNT. Purified nanotubes were obtained after a soft-bake at $250{ }^{\circ} \mathrm{C}$ for $24 \mathrm{~h}$ followed by sonicating in concentrated $\mathrm{HCl}$ at $80^{\circ} \mathrm{C}$ for $20 \mathrm{~min}$, and washing with water [22]. Functionalization $[19,20]$ was initiated by refluxing the SWNT at $115^{\circ} \mathrm{C}$ in $2.6 \mathrm{M}$ nitric acid for 12 or $48 \mathrm{~h}$ while stirring to decorate the SWNT with carboxylic acid groups (-COOH). After washing in water and drying, these nanotubes were suspended in dimethyl formamide (DMF) to which thionyl chloride $\left(\mathrm{SOCl}_{2}\right)$ was subsequently added and stirred at $70{ }^{\circ} \mathrm{C}$ for $24 \mathrm{~h}$ to transform the $-\mathrm{COOH}$ groups to chloric acid (-COCl). After washing with anhydrous THF and drying, the nanotubes were stirred in excess f12 (dodecylamine, $\mathrm{C}_{12} \mathrm{H}_{27} \mathrm{~N}$, Aldrich) at $95^{\circ} \mathrm{C}$ for $96 \mathrm{~h}$. Excess f12 was removed by ethanol washing. The same procedure was used for functionalization by f18 (octadecylamine, $\mathrm{C}_{18} \mathrm{H}_{39} \mathrm{~N}$, Acros Organics). The surfactant NaDDBS (dodecylbenzenesulfonic acid [sodium salt], $\mathrm{C}_{18} \mathrm{H}_{29} \mathrm{NaO}_{3} \mathrm{~S}$, Aldrich) was used in 10:1 weight ratio with respect to the SWNT [17]. 
Interfacial Polymerization of Neat Nylon 6,6: The polymerization system consists of a toluene phase containing the dichloric acid adipoyl chloride $\left(\mathrm{C}_{6} \mathrm{H}_{8} \mathrm{Cl}_{2} \mathrm{O}_{2}\right.$, Aldrich) and a water phase containing the diamine 1,6-hexamethylene diamine $\left(\mathrm{C}_{6} \mathrm{H}_{16} \mathrm{~N}_{2}\right.$, Fluka) and the base sodium hydroxide (Fisher Scientific). For the polymerization of the neat nylon 6,6, equimolar monomer solutions of 0.0244 moles were made with $4 \mathrm{ml}$ adipoyl chloride in $150 \mathrm{ml}$ toluene and $3.37 \mathrm{ml} \mathrm{1,6-hexamethylene} \mathrm{diamine} \mathrm{in} 300 \mathrm{ml}$ deionized water, which also contained 0.049 moles sodium hydroxide. The solutions were combined in a blender (Waring, model 51BL31) and reacted for five minutes with agitation. The obtained nylon 6,6 was filtered through a Büchner fritted disk funnel and washed repeatedly with water, acetone, and toluene. After washing, the nylon 6,6 was dried at $80{ }^{\circ} \mathrm{C}$ for $20 \mathrm{~h}$.

In Situ Polymerization of Nylon 6,6 with SWNT: The in situ polymerization of nylon 6,6 in the presence of the nanotubes was performed with the same reagent ratios as described above for the neat nylon 6,6. The SWNT were either suspended in toluene (purified SWNT and functionalized SWNT with $-\mathrm{COOH},-\mathrm{f12}$, or -f18) or in water (NaDDBS stabilized SWNT). Composites of $3.5-5.5 \mathrm{wt} \%$ SWNT were obtained, depending on the nanotube - monomer ratio. The SWNT weight without the functional groups was used for the $\mathrm{wt} \%$ calculation. The yield of the in situ nylon 6,6 polymerization ( $\sim 5 \%$ ) was unaffected by the addition of SWNT. To adjust the nanotube loading, the SWNT / nylon composites were dissolved in formic acid, along with commercial nylon 6,6 ( $\bar{M}_{v}=22,000 \mathrm{~g} / \mathrm{mol}$, Scientific Polymer Products $)$ and precipitated in water. The following composites were made by this method: $2 \mathrm{wt} \%$ for the purified SWNT, SWNT-COOH, SWNT-f12, and SWNT-NaDDBS; $1.6 \mathrm{wt} \%$ for the SWNT-f18.

Processing and Characterization: Characterization of the functionalized SWNT was performed with a Perkin-Elmer 2000 FTIR spectrometer, a Renishaw micro-Raman spectrometer, and an SDT 2960 DTA/TGA analyzer from TA Instruments. Functionalized SWNT were deposited from solution on a ZnSe ATR crystal for FTIR measurements. The distribution of the nanotubes in solvent suspensions prior to the in 
situ polymerization was determined by optical transmission microscopy of the suspensions.

The viscosity average molecular weight of nylon 6,6 was determined by capillary viscosimetry using an Ubbelohde viscometer with a kinematic viscosity constant of 0.003 $\mathrm{mm}^{2} / \mathrm{s}^{2}$ (type 0C from Cannon Instrument Co.). The efflux time of nylon 6,6 solutions in $90 \%$ formic acid was measured at concentrations from 2.23 to $10.41 \mathrm{mg} / \mathrm{ml}$ at $25{ }^{\circ} \mathrm{C}$. The relative viscosity, which is proportional to the efflux time, was plotted as a function of concentration; the intrinsic viscosity was then determined from this plot using the Huggins and Kramer equations.

Films of SWNT / nylon 6,6 composites ( $150 \mu \mathrm{m}$ thickness) were prepared by hot pressing at $270{ }^{\circ} \mathrm{C}$ and used to characterize the nanotube dispersion by optical transmission microscopy. Rods were extruded at $275^{\circ} \mathrm{C}$ with a DACA SpinLine consisting of a single spinneret hole $(500 \mu \mathrm{m})$ attached to a heated barrel with a piston extruder. Fibers were extruded at $275^{\circ} \mathrm{C}$ and drawn under tension with a variable-speed winder.

The percent crystallinities of the nylon 6,6 and SWNT / nylon 6,6 composites were determined using a Perkin-Elmer differential scanning calorimeter (DSC 7) at a heating rate of $10 \% \mathrm{~min}$. Tensile tests on fibers were conducted on a Instron 4206 with a gauge length of $2.54 \mathrm{~cm}$ and a cross-head speed of $10 \mathrm{~mm} / \mathrm{min}$. Electrical conductivity was measured in a two probe configuration on extruded rods with a Raman intensity ratio $\left(I_{0^{\circ}} / I_{90^{\circ}}\right)$ of $\sim 1.8$, corresponding to a Lorentzian FWHM of $125^{\circ}[23,24]$.

\section{Results and Discussion}

Interfacial Polymerization of Nylon 6,6: The reaction product of the interfacial polymerization is a white powder. Fourier-transform IR confirmed the chemical structure of the nylon 6,6, showing absorptions for all required chemical groups: N-H stretch at $3304 \mathrm{~cm}^{-1}$, C-H stretch at 2860-2940 $\mathrm{cm}^{-1}$, Amide-I at $1632 \mathrm{~cm}^{-1}$, and Amide-II at 1540 $\mathrm{cm}^{-1}[25]$. The Mark-Houwink equation ([$\left.\eta\right]=K_{v} M_{v}^{a}$ using $K_{v}=3.53 \mathrm{E}-4$ and $a=0.786$, for nylon 6,6 in $90 \% \mathrm{HCOOH}$ at $25^{\circ} \mathrm{C}$ [25]) was used to determine the viscosity 
averaged molecular weight $\overline{M_{v}}$ from the intrinsic viscosity measurements, resulting in a $\overline{M_{v}} \sim 35,000 \mathrm{~g} / \mathrm{mol}$.

During processing at elevated temperatures, such as hot pressing or melt fiber spinning, the nylon 6,6 darkened in color and became brittle. Acidic reaction products that were not completely removed in the washing steps can catalyze thermal oxidation, which is the most probably cause for the observed degradation. The interfacially polymerized nylon 6,6 was blended with commercial nylon 6,6 to stabilize the system and to enhance hot pressing and melt fiber spinning. The melting temperature $\left(261{ }^{\circ} \mathrm{C}\right)$ and the total crystallinity ( $29 \%$ ) did not change upon blending.

SWNT Functionalization: The characterization of the SWNT after functionalization was carried out by FTIR, Raman, and TGA analysis. After nitric acid treatment SWNT show an IR peak at $1727 \mathrm{~cm}^{-1}$ (Fig. 1b), indicative of the $\mathrm{C}=\mathrm{O}$ stretching vibration of carboxylic acid groups [19]. The broad peak between $3000-3600$ $\mathrm{cm}^{-1}$ is assigned to the $\mathrm{O}-\mathrm{H}$ stretches, while the peak at $1585 \mathrm{~cm}^{-1}$ is attributed to the SWNT C $=$ C stretching mode associated with sidewall attachment [26].

Raman spectra provide further evidence of $-\mathrm{COOH}$ functionalization of the SWNT (Fig. 2). The typical radial breathing (RBM) and tangential (G) modes for HiPco SWNT exhibit peaks at $200-263$ and $\sim 1591 \mathrm{~cm}^{-1}$, respectively, and are observed here for both the pristine and nitric acid treated SWNT. The disorder mode at $1292 \mathrm{~cm}^{-1}$, attributed to $\mathrm{sp}^{3}$-hybrized carbon in the hexagonal framework of the SWNT walls and amorphous carbon, increases for the nitric acid treated SWNT as more -COOH groups are covalently attached to the nanotube walls $[27,28]$. Also, the G-mode of SWNT$\mathrm{COOH}$ is shifted upward from $1591 \mathrm{~cm}^{-1}$ to $1593 \mathrm{~cm}^{-1}$ as compared to pristine SWNT. The - $\mathrm{COOH}$ groups can act as electron acceptors which results in p-doping of the SWNT that causes the C-C bonds to stiffen $[29,30]$.

After transforming the carboxylic acid to chloric acid and reacting with $\mathrm{f} 12$ and f18, the FTIR spectra shows a new broad peak at $1668 \mathrm{~cm}^{-1}$ (Fig. 1c), suggesting the formation of an amide linkage between the f12 or f18 and SWNT [20]. The C-H stretching mode peaks (2922 and $2852 \mathrm{~cm}^{-1}$ ) are greatly enhanced by the alkyl chains, and the peak at $1467 \mathrm{~cm}^{-1}$ is assigned to the $\mathrm{C}-\mathrm{H}$ bending mode. The FTIR and Raman 
measurements effectively show that SWNT with - $\mathrm{COOH}$ functional groups was produced during the $\mathrm{HNO}_{3}$ treatment and subsequently converted to functionalize SWNT as evidenced by the amide group observed via FTIR.

The degree of functionalization, i.e. the percentage of carbon atoms in SWNT that are covalently bonded to a side chain, was estimated from the two-step weight loss in TGA experiments (Fig. 3). Alkyl chains f12 and f18 attached to SWNT degrade primarily below $380{ }^{\circ} \mathrm{C}$, giving rise to a major peak at $\sim 300{ }^{\circ} \mathrm{C}$ in the derivative plot. The alkyl chain weight losses for SWNT-f12 and SWNT-18 are $~ 31$ and $\sim 43 \%$, respectively, from which the number of attached alkyl chains were calculated. The mole percentage of f12 and f18 side chains relative to the number of carbon atoms in SWNT is $\sim 3 \%$, which corresponds to the fraction of SWNT atoms with $-\mathrm{COOH}$ after the nitric acid treatment. (This calculation assumes that all the $-\mathrm{COOH}$ groups reacted with amine-terminated alkyl chains, which is expected due to the excess of reactant.) Our observation that $\sim 3 \%$ of the SWNT carbon atoms have been functionalized with $-\mathrm{COOH}$ is in good agreement with previous results from acid-base titration methods [31,32]. This result indicates a grafting density of $\sim 4$ alkyl chains per $1 \mathrm{~nm}$ of a $(10,10)$ SWNT. If the f12 or f1 8 chains lie on the SWNT surface, then the surface areas per chain are $\sim 0.13$ and $\sim 0.2 \mathrm{~nm}^{2}$, which corresponds to a surface coverage of only 2.9 and $4.4 \%$, respectively. Thus, both in terms of the number of SWNT carbon atoms involved and the surface coverage, the level of functionalization is quite modest.

SWNT Dispersion: Based simply on visual inspections, suspensions of purified SWNT and SWNT-COOH in toluene contain SWNT agglomerates and particles. This results in unstable and inhomogeneous suspensions. The short polar $\mathrm{COOH}$ groups do not support SWNT dispersion in either water or apolar organic solvents like toluene. The functionalization of the SWNT with $\mathrm{f} 12$ and f1 8 improves dispersion and suspendability in toluene. SWNT-f12 produces a stable black suspension with loose agglomerates, while SWNT-f18 disperses in toluene to form stable, homogenous, translucent suspensions with no visible agglomerates. The longer alkyl chain $\mathrm{f} 18$ has a better repulsion effect between the nanotubes than does $\mathrm{f} 12$, which prevents bundling due to van der Waals forces between the SWNT. Therefore, purified and functionalized SWNT were suspended in the 
toluene phase during the in situ polymerization. Nanotubes suspended in water were stabilized with NaDDBS and show dispersions comparable to SWNT-f18 in toluene; no agglomerates or particles are visible.

Nanotube dispersion in toluene and water, at the concentration used for the in situ polymerization $(0.35 \mathrm{mg} / \mathrm{ml}$ or $0.0004 \mathrm{wt} \%)$, is shown in more detail in the optical micrographs in Figure 4 (left column). Purified SWNT and SWNT-COOH in toluene are assembled in loose agglomerates surrounding dense particles. SWNT-f12 toluene suspensions show a much better dispersion with smaller, less dense agglomerates. SWNT-f18 suspensions are featureless on this length scale, suggesting uniformly suspended ropes or single nanotubes [17,20]. SWNT-NaDDBS suspended in water are well dispersed without any indications of particles or agglomerations.

The dispersion of nanotubes in composites after the in situ polymerization and blending with commercial nylon 6,6 is shown in the right column of Figure 4 . The dispersion of purified SWNT, SWNT-COOH, and -f12 in nylon 6,6 composites is comparable; dense agglomerates are visible in a more homogenous background. Agglomerates in the initial toluene suspension were not broken up during the in situ polymerization, but were instead preserved and compacted in the composite. The good dispersion of SWNT-f18 in toluene is maintained in the composite, resulting in a composite with a homogenous dispersion. SWNT-NaDDBS / nylon 6,6 composites show inhomogenities that arise during in situ polymerization and could stem from interactions between the monomers, the reaction products $(\mathrm{HCl})$ and the surfactant, or segregation of the surfactant to the water/toluene interface to produce agglomerates. The blending of the in situ polymerized SWNT / nylon 6,6 composites with commercial nylon 6,6 using the coagulation process with formic acid did not change the dispersion of SWNT in the composites. The nylon 6,6, which is dissolved during this step, supports the nanotubes in suspension.

Overall, well-dispersed nanotube suspensions produce nanocomposites with good nanotube dispersion via in situ polymerization. This in situ polymerization method can readily incorporate a variety of nanotubes suspended in toluene or water into nylon 6,6 . 
Composite Processing: The SWNT / nylon 6,6 composites prepared above were subsequently melt-spun into fibers, extruded into rods, and hot-pressed into films. The SWNT dispersion in melt-spun fibers and extruded rods is homogenous (Fig. 5a), with no apparent change in dispersion observed for any fiber or rod diameter. Existing SWNT agglomerates that are present after the in situ polymerization remain in the processed composites. In contrast, both homogeneous and heterogeneous SWNT dispersions were found in hot-pressed films depending on the time, temperature and pressure used during the pressing. SWNT-f1 8 / nylon 6,6 composite films pressed at a load of $3000 \mathrm{lbs}$. and cooled immediately show homogenous nanotube dispersion (Fig. 5b), while composites pressed at an elevated load of $5000 \mathrm{lbs}$. and held at $265^{\circ} \mathrm{C}$ for $2 \mathrm{~min}$ before cooling exhibit large isolated nanotube agglomerates (Fig. 5c).

This effect was further investigated by constraining the SWNT-f1 8 / nylon 6,6 composite between glass slides while heating in an optical microscope. The initial dispersion is homogenous upon heating (Fig. 6a). As the nanocomposite flows to fill the gap between the glass slides, the SWNT quickly flocculate $(30 \mathrm{sec})$ and then aggregate into distinct clusters $(180 \mathrm{sec})$ in flow-induced flocculation, Figure $6 \mathrm{c}$. In contrast, when the composite melt is kept tranquil by melting on a glass slide without cover glass, the SWNT dispersion remains homogenous over a long time period (10 min). Flow-induced flocculation also occurs in composites containing purified SWNT, SWNT-COOH, and SWNT-f12, but the flocculation is less dramatic because the initial composite dispersion is less homogeneous.

Lin-Gibson et al. have shown that MWNT in a Newtonian fluid can form agglomerates under a weak linear shear flow [33]. Similarly, the SWNT agglomeration in SWNT-f1 / nylon 6,6 composites observed here is caused by the weak shear flow between glass sides or hot press plates. Even weak shear forces are sufficient to induce SWNT-SWNT collisions that promote the growth of SWNT agglomerates by entrapping more nanotubes that finally result in the observed flocculation. The duration of the shear flow is also critical as suggested by the observation that agglomeration while hot pressing occurs only when the pressure is maintained for an extended period of time above the melting temperature before cooling. The shear flow during melt fiber spinning and 
extrusion exert larger shear forces that are able to break up any transient agglomerates that form due to SWNT-SWNT collisions. Furthermore, the duration of these extensional flows are very short $(>1 \mathrm{sec})$, and thus good dispersion is maintained during our extrusion processes.

The macroscopic appearance of melt-spun fibers is influenced by the SWNT characteristics, especially nanotube length and dispersion. Melt fiber spinning of pristine nylon 6,6 and SWNT-f12 and SWNT-f18 / nylon 6,6 composites results in fibers with a smooth surface and homogeneous radial diameter (Fig. 7). Conversely, purified SWNT / nylon 6,6 composite fibers have rough surfaces and irregular fiber diameters that indicate an increased melt viscosity. Linear viscoelastic studies have shown that SWNT / polymer composites can exhibit solid-like behavior [34], which would give rise the observed melt fraction in the nanocomposite fibers prepared with purified nanotubes.

The work of our group on the effect of nitric acid treatment on SWNT showed that refluxing HiPco SWNT in nitric acid gradually reduces the number of long nanotubes and increases the amount of short nanotube fragments as the reflux time increases [35]. SWNT that were used as SWNT-COOH were treated in nitric acid for 12 $\mathrm{h}$, while SWNT prepared for the $\mathrm{f} 12$ and $\mathrm{f} 18$ functionalization were treated for $48 \mathrm{~h}$. Purified and surfactant suspended SWNT were purified with $\mathrm{HCl}$ at short times (20 min), minimizing any damage or reduction in length. This implies that SWNT-f12 and SWNTf18 are the shortest, followed by SWNT-COOH, and that purified and surfactant suspended SWNT are the longest. Longer nanotubes are more likely to form a nanotube network and thereby induce solid-like behavior [34]. Thus, the long purified SWNT potentially increase the melt viscosity causing melt fracture that results in rough fiber surfaces and inhomogeneous diameters, whereas the shorter SWNT-12 and -18 do not markedly increase the composite melt viscosity, resulting in uniform fibers with smooth fiber surfaces.

Electrical Conductivity and Mechanical Properties: Multiple factors can increase the electrical conductivity in composites with conducting fillers: filler and matrix conductivities, filler concentration, filler dispersion, filler-filler contacts, filler shape, and filler orientation when the filler is anisotropic. For example, when nanotubes are 
unoriented and well dispersed as isolated nanotubes or small bundles, simple percolation theories predict that increasing the aspect ratio results in a percolation threshold at lower loadings. Conversely at a fixed loading and fixed level of orientation, composites with large aspect ratio fillers form more percolating pathways and thereby have higher electrical conductivity. Note that we have separately investigated the effect of nanotube alignment on electrical conductivity in polymer nanocomposites [36].

The electrical conductivity measurements on extruded composite rods and the tensile tests on melt-spun composite fibers show distinctive trends for the various SWNT fillers. The dependence of the nanotube loading is apparent for purified SWNT and SWNT-18 / nylon 6,6 composites (Fig. 8); the electrical conductivity is improved by approximately two orders of magnitude when the SWNT loading increases from $\sim 2$ to 5 $\mathrm{wt} \%$. At $2 \mathrm{wt} \% \mathrm{SWNT}$, the electrical conductivities of the SWNT / nylon 6,6 composite rods increases with SWNT length with SWNT-f12 the shortest, followed by SWNT$\mathrm{COOH}$, and purified and surfactant suspended SWNT being the longest. This highlights the importance of nanotube processing methods and the resulting nanotube length. These results are consistent with work by Bai et al. on MWNT of different lengths in an epoxy matrix [37], showing that longer nanotubes are more likely to build percolating paths than shorter nanotubes at a fixed nanotube loading. In addition, a reduction in the conductivity of the funcationalized nanotubes relative to the purified nanotubed might contribute to the reduced electrical conductivity at $2 \mathrm{wt} \% \mathrm{SWNT}$. Covalently attached sidegroups to the body of SWNT are defects, because the conjugated $\mathrm{sp}^{2}$ bonds are converted to saturated $\mathrm{sp}^{3}$, thereby weakening the electronic band structure of SWNT [38]. Presently, we cannot separate the two effects of nanotube length and nanotube conductivity.

SWNT-f12 and -f18 have presumably the same length but the electrical conductivity of SWNT-12 / nylon 6,6 composites is $\sim 3$ orders of magnitude higher than for SWNT-f1 8 / nylon 6,6 composites (at $1.6 \mathrm{wt} \% \mathrm{SWNT}$ ). The electrical conductivity in composites also depends on the ability of the electrons to transfer between adjacent nanotubes. Direct contact between nanotubes results in the highest probability for this event. The hydrophobic alkyl chains of SWNT-f12 and -f18 are more likely to interact with the nanotubes to which they are attached, than with the polar hydrophilic nylon 6,6. 
Thus, the functionalization of SWNT in the nylon 6,6 matrix constructs an electrically insulating partial layer around the nanotubes that reduces the number of direct nanotubenanotube contacts and increases the distance between nanotubes $[1,13]$. This partial layer reduces the probability of electron quantum tunneling [39] and consequently reduces the electrical conductivity of the composites. The longer f18 chains cover more of the nanotube surface than $\mathrm{f12}$, which correlates to a lower electrical conductivity in the composite. The electrical conductivity of SWNT-NaDDBS / nylon 6,6 composite is very close to composites with purified SWNT, despite the presence of surfactant molecules that potentially could act as an insulating layer. These results suggest that the surfactant molecules do not significantly hamper electron transfer, perhaps because the surfactant molecules are expelled from the nanotube-nanotube junctions and reassemble around the contact point. Obviously, grafted chains, as is the case in SWNT-f12 and SWNT-f18, do not have that liberty.

All composites except SWNT-f18 / nylon 6,6 show nanotube agglomerations but have higher electrical conductivities than SWNT-f18 / nylon 6,6, indicating a positive effect of nanotube agglomeration. Although it is generally assumed that improved nanotube dispersion enhances electrical conductivity as the formation of conducting paths though the composite is more probable with well dispersed nanotubes, it has been shown that the formation of macroscopic nanotube aggregates that connect to each other results in a lower percolation threshold as compared to well dispersed nanotubes [40,41]. The electrical conductivities of SWNT / polymer composites are influenced by a variety of filler attributes (length, functionalization, etc.) and processing conditions (loading, dispersion, etc.) that require careful investigation to fully optimize.

The elastic moduli obtained from tensile tests on melt-spun $\sim 2 \mathrm{wt} \% \mathrm{SWNT} /$ nylon 6,6 composite fibers increase with SWNT alignment (Fig. 9), that is towards smaller fiber diameters. This agrees our previous work on SWNT / HDPE composite fibers showing that SWNT alignment increases the elastic modulus at fixed loadings [11]. Figure 9 also provides evidence that the elastic moduli of nanotube / nylon composites are enhanced by improved dispersion, and higher nanotube aspect ratios. For example, purified SWNT / nylon 6,6 composite fibers show an enhanced modulus of $\sim 30 \%$ 
relative to composites with either SWNT-f12 or -f18 / nylon 6,6 fibers with similar fiber diameter $(\sim 120 \mu \mathrm{m})$. We attribute this improvement to the longer length and higher aspect ratios of purified SWNT in this study. In fact, the shorter SWNT-f12 and -f18 show moduli similar to nylon 6,6 fibers.

The fracture toughness of nylon 6,6 fibers is similar to that of SWNT-f18 / nylon 6,6 composites fibers, but the composite fibers with purified SWNT and SWNT-f12 show a decrease in fracture toughness of $\sim 2$ and 3 orders of magnitude, respectively. This implies that good SWNT dispersion is critical for improved fracture toughness, because SWNT agglomerates might act as structural defects and cause the fiber to fracture immediately after yielding.

Effective mechanical reinforcement in SWNT-polymer composites can be achieved with nanotubes with large length and good dispersion. The SWNT / nylon 6,6 composites in this work have either good dispersion (SWNT-f18) or large length (purified SWNT), but not both. More careful SWNT treatment prior to composite fabrication to maintain nanotube length and improve dispersion in composites promises improved mechanical properties. SWNT sidewall functionalization with alkyl chains that have a positive affinity for the nylon 6,6 matrix might also lead to high performance composites.

\section{Conclusions}

We have adapted an interfacial in situ polymerization method to the fabrication of SWNT / nylon 6,6 nanocomposites. This versatile fabrication method incorporates SWNT suspended in either water or toluene, and can be readily extended to a variety of nanofillers with different surface properties and solvent preferences. The quality of the nanofiller suspension prior to the interfacial polymerization determines to a large extent the nanofiller dispersion in the resulting nanocomposite. In the case of SWNT, functionalizing with alkyl chains promotes the suspension of SWNT and subsequently improves dispersion in the composites. Using surfactants to disperse nanotubes is less reliable during the two-phase in situ polymerization. 
The properties of the various SWNT - nylon 6,6 composites were explored. Notably, low shear forces in these composites can cause nanotube agglomeration, and therefore must be considered during nanocomposite processing. SWNT characteristics in

polymer composites have a tremendous influence on both the electrical conductivity and mechanical properties. The influence of various SWNT cannot be attributed to a single nanotube parameter; rather, it is necessary to consider multiple SWNT characteristics, including nanotube length, dispersion, and type and degree of the nanotube functionalization. Composites containing purified SWNT that maintain their length but exhibit poor dispersion have an increase in elastic modulus and electrical conductivity. The functionalization of the SWNT with -f12 and -f18 improves nanotube dispersion, but the reduced nanotube length and larger nanotube separation resulting from functional groups limits mechanical and electrical properties of the composites containing these nanotubes.

\section{Acknowledgements}

Funding for this research was provided by the National Science Foundation (DMR-MRSEC 05-20020 and DMR-IMR) and the Office of Naval Research (N0001403-1-0890 and DURINT N00014-00-1-0720). 


\section{References}

1. Shaffer MSP; Windle AH. Advanced Materials 1999;11(11):937.

2. Safadi B; Andrews R; Grulke EA. Journal of Applied Polymer Science 2002;84(14):2660.

3. Du F; Fischer JE; Winey KI. Journal of Polymer Science: Part B: Polymer Physics 2003;41:3333.

4. Qian D; Dickey EC; Andrews R; Rantell T. Applied Physics Letters 2000;76(20):2868.

5. Pötschke P; Bhattacharyya AR; Janke A. European Polymer Journal 2004;40:137.

6. Andrews R; Jacques D; Minot M; Rantell T. Macromolecular Materials Engineering 2002;287(9):395.

7. Zhang WD; Shen L; Phang IY; Liu T. Macromolecules 2004;37:256.

8. Sandler JKW; Pegel S; Cadek M; Gojny F; van-Es M; Lohmar J; Blau WJ; Schulte K; Windle AH; Shaffer MSP. Polymer 2004;45:2001.

9. Liu T; Phang IY; Shen L; Chow SY; Zhang WD. Macromolecules 2004;37:7214.

10. Sennett M; Welsh E; Wright JB; Li WZ; Wen JG; Ren ZF. Applied Physics A $2003 ; 76: 111$.

11. Haggenmueller R; Zhou W; Fischer JE; Winey KI. Journal of Nanoscience and Nanotechnology 2003;3(1):105.

12. Park C; Ounaies Z; Watson KA; Crooks RE; Smith J; Lowther SE; Connell JW; Siochi EJ; Harrison JS; St.Clair TL. Chemical Physics Letters 2002;364:303.

13. Barraza HJ; Pompeo F; Orear EA; Resasco DE. Nano Letters 2002;2(8):797.

14. Gao J; Itkis ME; Yu A; Bekyarova E; Zhao B; Haddon RC. Journal of the American Chemical Society 2005;127:3847.

15. Kumar S; Dang TD; Arnold FE; Bhattacharyya AR; Min BG; Zhang X; Vaia RA; Park C; Adams WW; Hauge RH; Smalley RE; Ramesh S; Willis PA. Macromolecules 2002;35(24):9039.

16. Moniruzzaman M; Du F; Romero N; Winey KI. Polymer 2005;in press.

17. Islam MF; Rojas E; Bergey DM; Johnson AT; Yodh AG. Nano Letters 2003;3(2):269. 
18. O'Connell MJ; Boul P; Ericson LM; Huffman C; Wang Y; Haroz E; Kuper C; Tour J; Ausman KD; Smalley RE. Chemical Physics Letters 2001;342:265.

19. Chen J; Hamon MA; Hu H; Chen Y; Rao AM; Eklund PC; Haddon RC. Science 1998;282:95.

20. Hamon MA; Chen J; Hu H; Chen Y; Itkis ME; Rao AM; Eklund PC; Haddon RC. Advanced Materials 1999;11(10):834.

21. Nikolaev P; Bronikowski MJ; Bradley RK; Rohmund F; Colbert DT; Smith KA; Smalley RE. Chemical Physics Letters 1999;313:91.

22. Zhou W; Ooi YH; Russo R; Papanek P; Luzzi DE; Fischer JE; Bronikowski MJ; Willis PA; Smalley RE. Chemical Physics Letters 2001;350:6.

23. Haggenmueller R; Gommans HH; Rinzler AG; Fischer JE; Winey KI. Chemical Physics Letters 2000;330:219.

24. Hwang J; Gommans HH; Ugawa A; Tashiro H; Haggenmueller R; Winey KI; Fischer JE; Tanner DB; Rinzler AG. Physical Review B 2000;62(20):R13310.

25. Kohan MI, Nylon Plastics. ed.; John Wiley \& Sons, Inc.: 1973; 'Vol.' p.

26. Zhu J; Peng H; Rodriguez-Macias F; Margrave JL; Khabashesku VN; Imam AM; Lozano K; Barrera EV. Advanced Functional Materials 2004;14(7):643.

27. Stevens JL; Huang AY; Peng H; Chiang IW; Khabashesku VN; Margrave JL. Nano Letters 2003;3(3):331.

28. Ying Y; Saini RK; Liang F; Sadana AK; Billups WE. Organic Letters 2003;5(9):1471.

29. Rao AM; Eklund PC; Bandow S; Thess A; Smalley RE. Nature 1997;388:257.

30. Claye A; Rahman S; Fischer JE; Sirenko A; Sumanasekera GU; Eklund PC. Chemical Physics Letters 2001;33:16.

31. Hamon MA; Hu H; Bhowmik P; NIyogi S; Zhao B; Itkis ME; Haddon RC. Chemical Physics Letters 2001;347:8.

32. Hu H; Bhowmik P; Zhao B; Hamon MA; Itkis ME; Haddon RC. Chemical Physics Letters 2001;345:25.

33. Lin-Gibson S; Pathak JA; Grulke EA; Wang H; Hobbie EK. Physical Review Letters 2004;92(4):048302-1. 
34. Du F; Scogna RC; Zhou W; Brand S; Fischer JE; Winey KI. Macromolecules 2004;37(24):9048.

35. Brand S; Du F; Haggenmueller R; Winey KI. in preparation.

36. Du F; Fischer JE; Winey KI. Physical Review B 2005;72:121404.

37. Bai JB; Allaoui A. Composites Part A: Applied Science and Manufacturing 2003;34:689.

38. Kamaras K; Itkis ME; H. Hu; Zhao B; Haddon RC. Science 2003;30:1501.

39. Ounaies Z; Park C; Wise KE; Siochi EJ; Harrison JS. Composites Science and Technology 2003;63:1637.

40. Sandler JKW; Kirk JE; Kinloch IA; Shaffer MSP; Windle AH. Polymer 2003;44:5893.

41. Nogales A; Broza G; Roslaniec Z; Schulte K; Sics I; Hsiao BS; Sanz A; GarciaGutierrez MC; Rueda DR; Domingo C; Ezquerra TA. Macromolecules 2004;37:7669. 


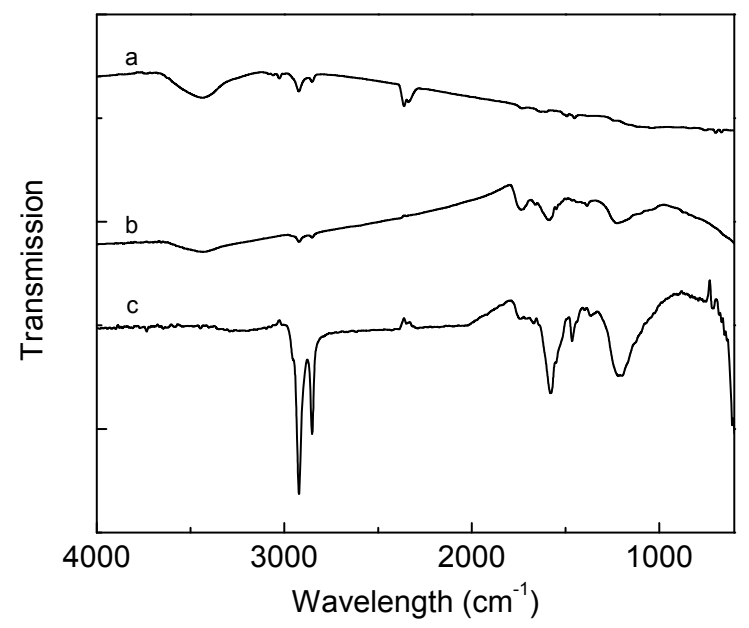

Figure 1: ATR-FTIR spectra of (a) pristine SWNT, (b) SWNT-COOH, (c) SWNT-f18. 


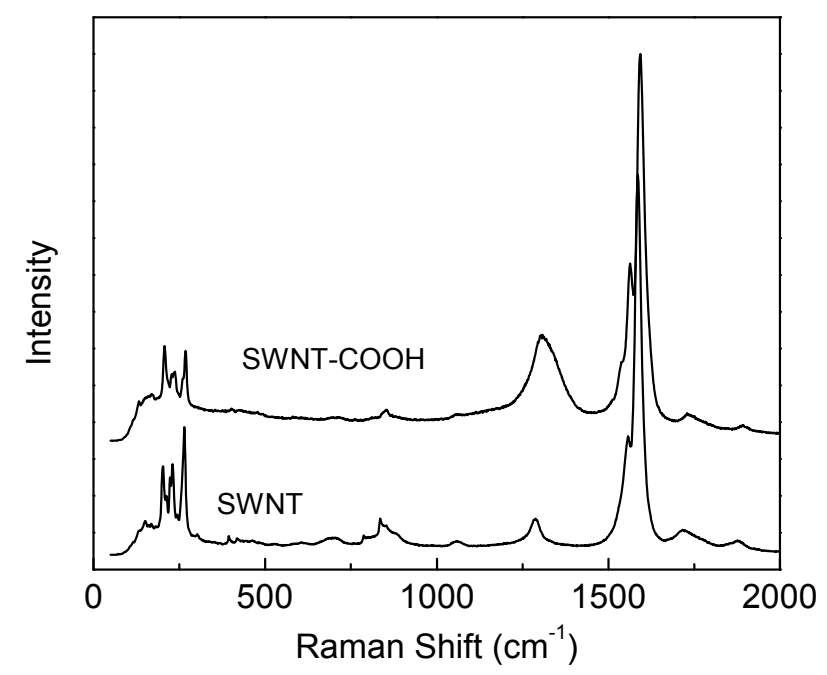

Figure 2: Raman spectra of pristine SWNT and nitric acid treated SWNT forming SWNT-COOH. The disorder bands at $1292 \mathrm{~cm}^{-1}$ are attributed to $\mathrm{sp}^{3}$-hybrized carbon in the hexagonal framework of the SWNT walls indicative of covalent attachment. The Gband at $1591 \mathrm{~cm}^{-1}$ is shifted upwards for the SWNT-COOH. 


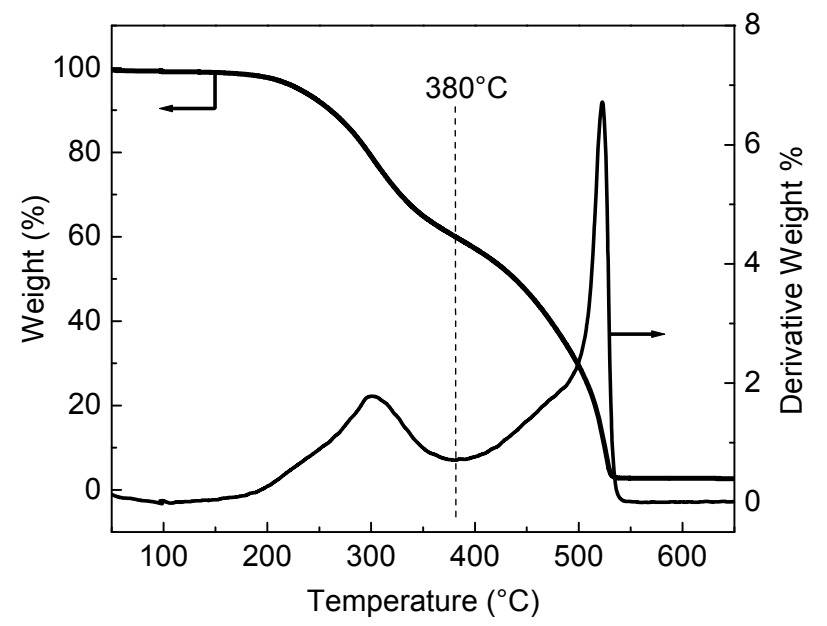

Figure 3: Thermal gravimetric analysis data for SWNT - f18 in air at a heating rate of 5 $\%$ min. 

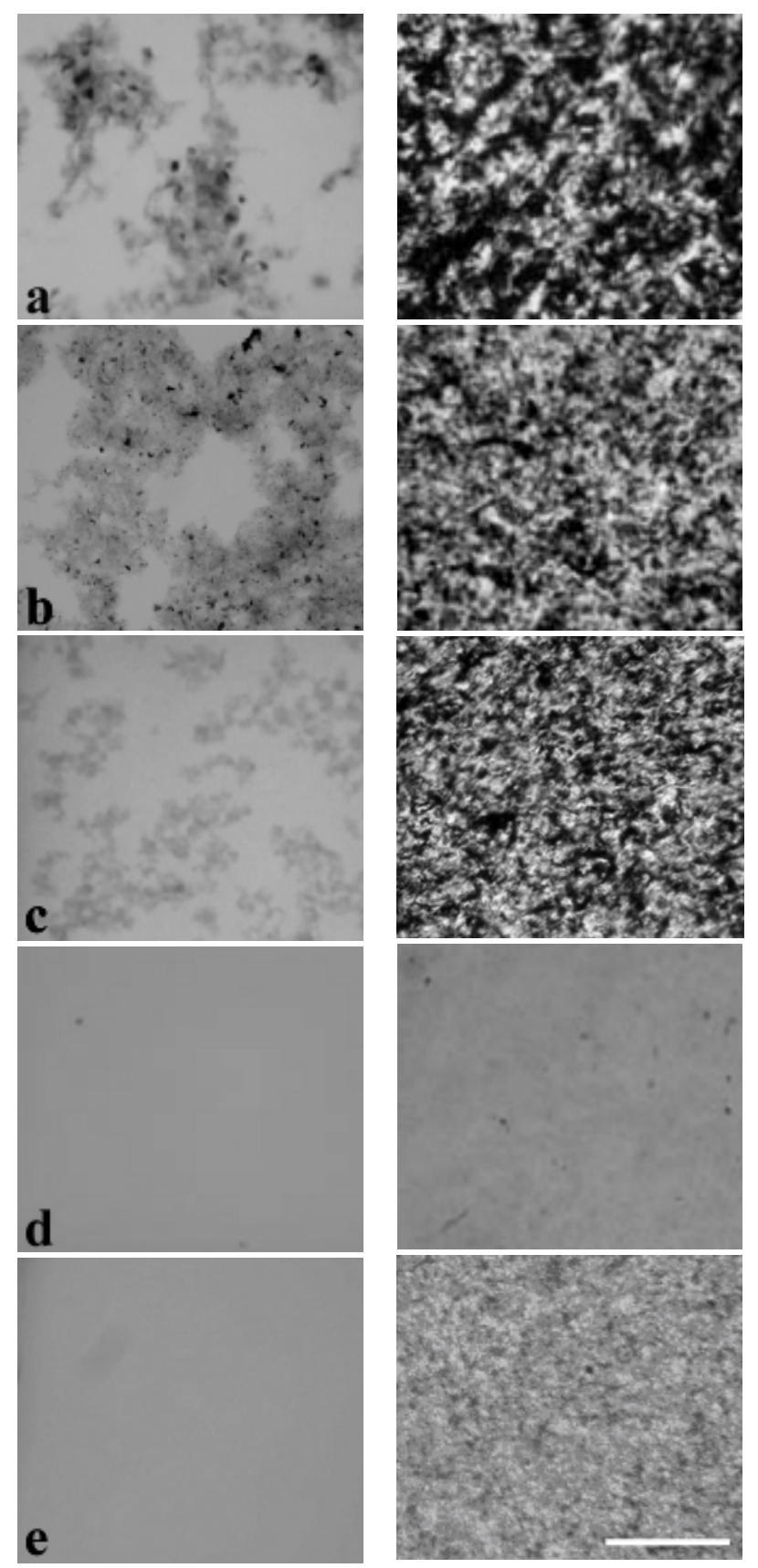

Figure 4: Left: Optical transmission micrographs of various SWNT suspensions at 0.35 $\mathrm{mg} / \mathrm{ml}$ : (a) purified SWNT in toluene, (b) SWNT-COOH in toluene, (c) SWNT-f12 in toluene, (d) SWNT-f18 in toluene, (e) SWNT-NaDDBS in water. Right: Optical transmission micrographs of hot-pressed SWNT / nylon 6,6 composites films at $2 \mathrm{wt} \%$ SWNT (1.6 wt\% SWNT-f18) prepared by in situ polymerization using the suspensions displayed on the left. Scale bar is $100 \mu \mathrm{m}$. 

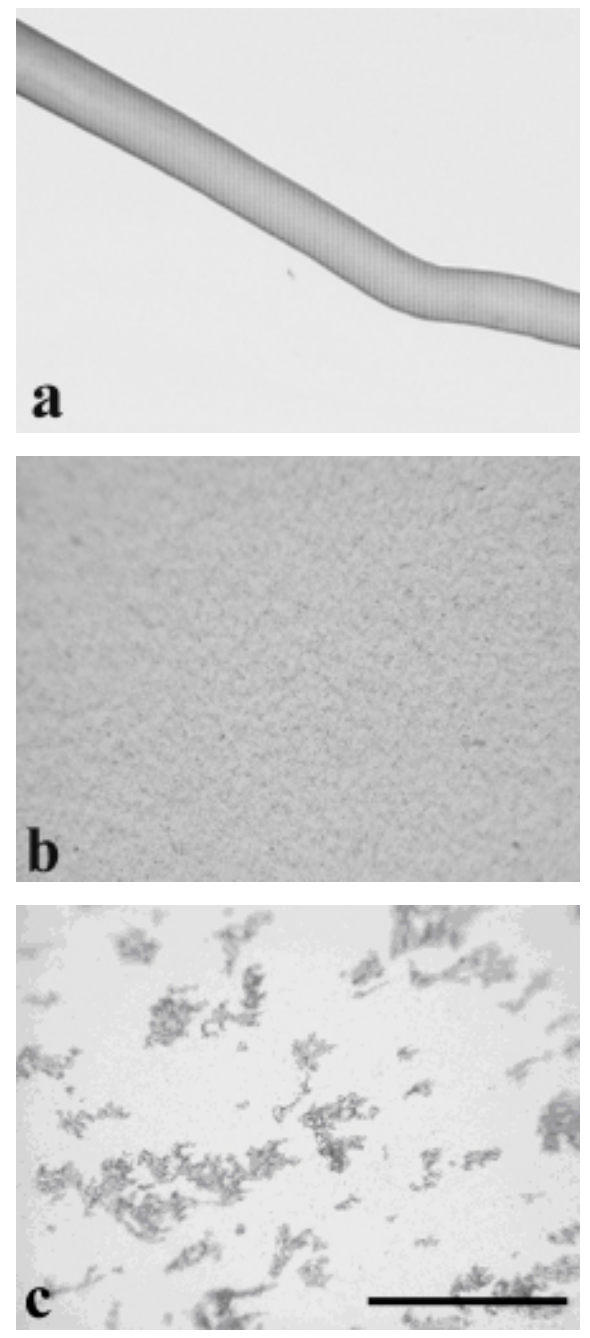

Figure 5: Optical transmission micrographs of $1.6 \mathrm{wt} \%$ SWNT-f18 / nylon 6,6 composites: (a) melt-spun fiber heated on a glass slide, (b) film hot pressed at $265^{\circ} \mathrm{C}$ and $3000 \mathrm{lbs}$. and immediately cooled, (c) film hot pressed at $265^{\circ} \mathrm{C}$ and $5000 \mathrm{lbs}$. and held for $2 \mathrm{~min}\left(265^{\circ} \mathrm{C}\right)$ before cooling. Scale bar is $100 \mu \mathrm{m}$. 

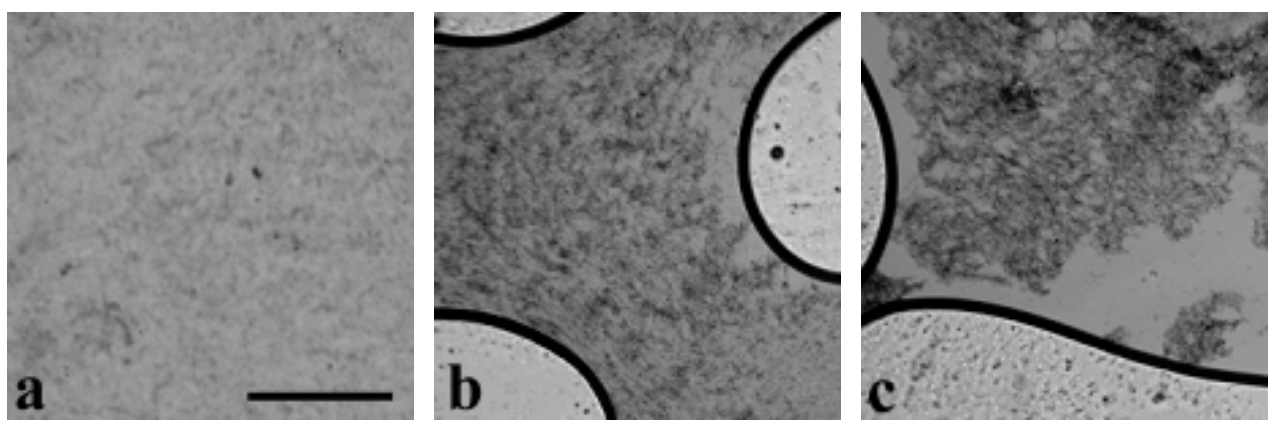

Figure 6: Optical tramission micrographs of the SWNT-f18 / nylon 6,6 composite while heated at $262{ }^{\circ} \mathrm{C}$ at various times. Composite is confined between a glass slide and a cover slip for (a) 0, (b) 30, and (c) 180 seconds. The dark curves in (b) and (c) indicate boundaries between air and the composite melt. Scale bar is $100 \mu \mathrm{m}$. 


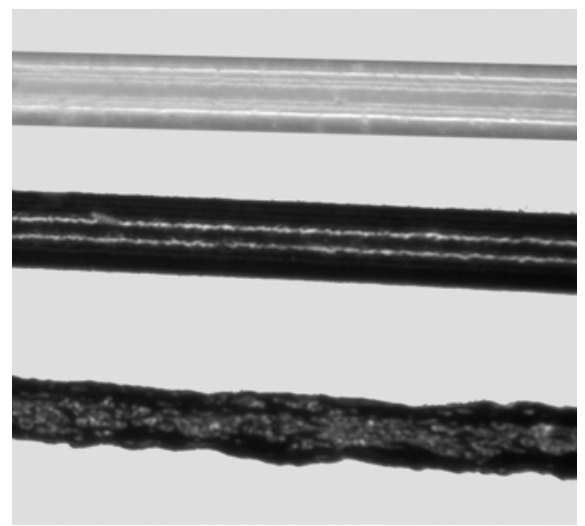

Figure 7: Optical micrographs of melt-spun fibers: (top) nylon 6,6, (middle) $1.6 \mathrm{wt} \%$ SWNT-f18 / nylon 6,6, and (bottom) $2 \mathrm{wt} \%$ purified SWNT / nylon 6,6. Fiber diameters are $\sim 50 \mu \mathrm{m}$. 


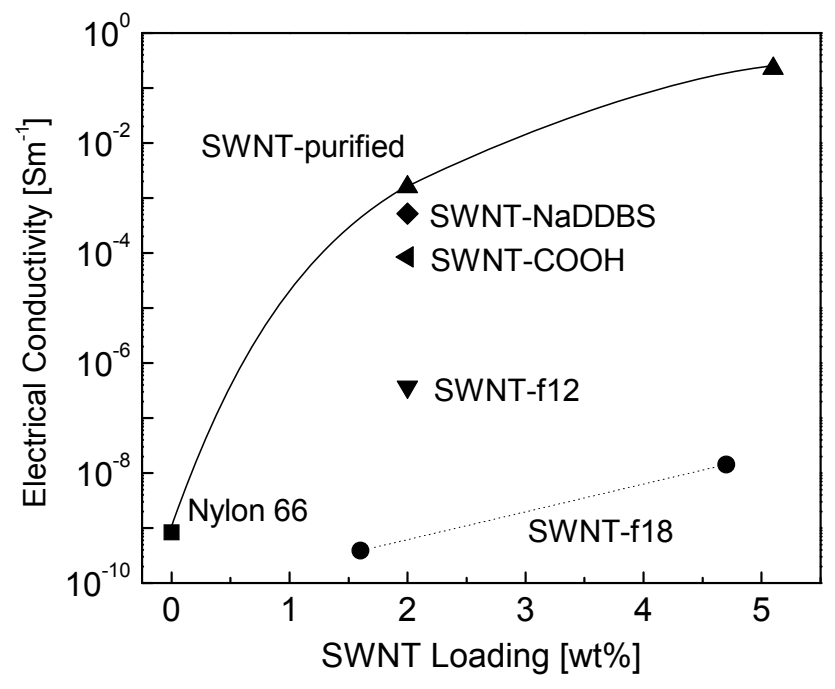

Figure 8: Electrical conductivity of extruded composite rods measured by a two-point probe configuration. Data points are the average of 3 individual measurements. 


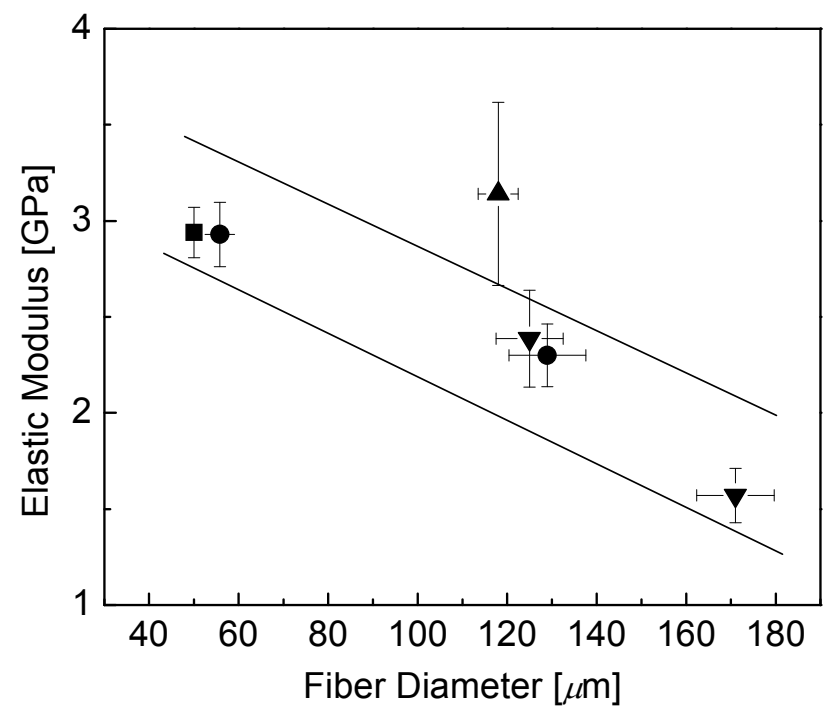

Figure 9: Elastic modulus from tensile tests on individual melt-spun fibers at a deformation rate of $10 \mathrm{~mm} / \mathrm{min}$. (-) nylon 6,6, ( $\mathbf{\Delta}) 2 \mathrm{wt} \%$ purified SWNT / nylon 6,6,

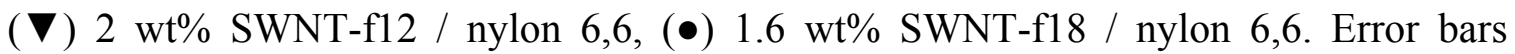
represent standard deviation of fiber diameter and elastic modulus. The large variation in modulus for the $2 \mathrm{wt} \%$ purified SWNT / nylon 6,6 composite fibers is associated with the inhomogeneous fiber diameter along the gauge length and inhomogeneous SWNT dispersion. 